\title{
A level set-based Eulerian approach for anisotropic wave propagation
}

\author{
Jianliang Qian ${ }^{\mathrm{a}}$, Li-Tien Cheng ${ }^{\mathrm{b}, *}$, Stanley Osher ${ }^{\mathrm{c}}$ \\ a Department of Mathematics, University of California, Los Angeles, CA 90095-1555, USA \\ ${ }^{\mathrm{b}}$ Department of Mathematics, University of California at San Diego, La Jolla, CA 92093-0112, USA \\ ${ }^{\mathrm{c}}$ Level Set Systems Inc., 1058 Embury Street, Pacific Palisades, CA 90272, USA
}

Received 2 November 2001; received in revised form 1 November 2002; accepted 4 November 2002

\begin{abstract}
The geometric optics approximation to high frequency anisotropic wave propagation reduces the anisotropic wave equation to a static Hamilton-Jacobi equation. This equation is known as the anisotropic eikonal equation and has three different coupled wave modes as solutions. We introduce here a level set-based Eulerian approach that captures all three of these wave propagations. In particular, our method is able to accurately reproduce the quasi-transverse, or quasi-S, waves with cusps, which form a class of multi-valued solutions. The level set formulation we use is borrowed from one for moving curves in three spatial dimensions, with the velocity fields for evolution following from the method of characteristics on the anisotropic eikonal equation. We present here our derivation of the algorithm and numerical results to illustrate its accuracy in different cases of anisotropic wave propagations related to seismic imaging.
\end{abstract}

(C) 2002 Elsevier Science B.V. All rights reserved.

\section{Introduction}

Geometric optics-based high frequency approximations provide an efficient framework as well as a set of tools for capturing the most singular part of a wave field [20], making it a fundamental assumption for modern seismic data processing such as Kirchoff migration [5,6], tomographic velocity analysis [43], and inversion $[8,12]$. The most singular part of a wave field is characterized by two quantities, the phase, or traveltime, function which satisfies the so-called eikonal equation and the amplitude function which satisfies a transport equation. The efficient computation of these two functions is thus of great practical importance in numerous areas such as oil industry applications $[13,14,30,32,45,50]$. Aside from wave applications, eikonal equations also appear in the calculus of variations, computer vision, image processing, and differential games, to name a few.

For isotropic wave propagations, the eikonal equations, which are of Hamilton-Jacobi form, are usually solved using viscosity solution-based solvers over spatial space [15,21,37,38,48,49], producing single-valued traveltime fields representing first-arrival traveltimes [22]. The relationship between traveltimes and geometric optics in an isotropic medium is that the rays are the trajectories orthogonal to the wavefronts, level sets of the traveltimes [11].

\footnotetext{
* Corresponding author.

E-mail addresses: qian@math.ucla.edu (J. Qian), lcheng@math.ucsd.edu (L.-T. Cheng), sjo@levelset.com (S. Osher).
} 
Moreover, if the medium is heterogeneous, there is a high probability that more than one ray will occupy any given spatial point, resulting in a multi-valued traveltime field over spatial space [51]. Nevertheless, the multi-valued traveltime field is essential, for example, in obtaining high resolution seismic images [23]. The Lagrangian approach to this problem is to use the method of characteristics to trace rays in phase space, thus taking care of multi-valuedness $[10,30]$. However, because in reality only a finite number of rays can be traced, Lagrangian methods in general produce nonuniform distributions of the traveltime field. The solution to this is interpolation, which can be cumbersome [30,50]. Therefore, Eulerian approaches are much desired for solving eikonal equations and, consequently, much research has been devoted to this. For example, Benamou in [2] proposed "big ray" tracing by localizing Riemann solvers in regions bounded by selected rays. Later on, in [3], he also enacted direct resolution of multi-valued phase space solutions of Hamilton-Jacobi equations. In [42], Symes derived a slowness matching finite difference method for computing multi-valued traveltimes by patching together local single-valued solutions of the eikonal equation via Fermat's principle into a global multi-valued traveltime field; see also [44]. In [16,17], Engquist and coworkers used the segment projection method to reparametrize multi-valued wavefronts into single-valued segments existing in a different space. Steinhoff et al., in [41], introduced an Eulerian method for capturing short acoustic pulses which allows the pulses to pass through each other. Related to this work, Ruuth et al. in [36] proposed a fixed grid method for capturing self-intersecting interfaces. More recently, in [26], Osher et al. introduced a phase space-based level set and Eulerian framework for constructing wavefronts that automatically handles multi-valued solutions when they appear. Finally, in regard to acoustic caustics, see the work of Benamou and Solliec [4] for more. All this effort to deal with multi-valued solutions, however, is for the isotropic case only. In this paper, we extend the level set-based Eulerian approach in [26] to apply to anisotropic wave propagations.

Because most sedimentary rocks in which oil reservoirs lie are anisotropic in the sense that the incident wave velocity depends on the incident angles, maximal resolution for imaging requires that the high frequency approximation of the wave field take into account the anisotropy of the wave propagation [12,13,47]. In general, 21 elastic parameters are needed to completely characterize an anisotropic medium. This can lead to very complicated wave propagations [18,24]. The high frequency approximation applied to the anisotropic wave equation gives rise to the Christoffel equation [24], and the related Christoffel determinant set equal to zero gives the so-called anisotropic eikonal equation. This equation characterizes a slowness surface which consists of three sheets corresponding to three different wave modes, the so-called quasi-P, quasi-SV, and quasi-SH waves [24], sometimes abbreviated to $\mathrm{qP}, \mathrm{qSV}$, and qSH, respectively.

Among the three sheets, the innermost one corresponds to the quasi-P waves and is always convex and seldom touches the others. The other two sheets may intersect with each other and the one associated to the quasi-SV waves may not be convex, leading to cusps and multi-valued wavefronts during wave propagation. To accurately solve the anisotropic eikonal equation, which takes the form of a static Hamilton-Jacobi equation, in an Eulerian framework, one technique is to use the level set method to obtain an evolution equation in artificial time [25,27] that can then be discretized using high order Runge-Kutta and essentially nonoscillatory schemes [19,28]. Efficiency can be achieved through localization of the algorithm around the zero level set denoting the desired wavefront $[1,29]$. Since this approach involves solving Hamilton-Jacobi equations in spatial space, it can only produce single-valued viscosity solutions. Other Eulerian approaches encounter the same difficulty and thus must limit themselves to the computation of single-valued wavefronts. Observing that the quasi-P slowness sheet is convex, Qian and Symes introduced a paraxial formulation to produce an evolution equation that uses one of the spatial variables for artificial time, with the resultant solution single-valued and giving the first-arrival traveltime field [31,33]. Also, Sethian and Vladimirsky have developed so-called ordered upwind schemes for static Hamilton-Jacobi equations which also produce single-valued traveltimes [39]. However, the quasi-S wavefronts and related multi-valued solutions are just as important as the quasi-P wavefronts for seismic imaging $[8,13]$ and thus, an efficient and accurate method for computing them is much needed. To our knowledge, so far, in the framework of the geometric optics approximation, the only approach for computing quasi-S wavefronts with cusps is the method of characteristics [9]. Thus, our work here is the first attempt of an Eulerian approach to this problem. 
In Section 2, we briefly give some background on the high frequency approximation of anisotropic wave propagations and on three coupled wave propagations. In Section 3, we consider anisotropic wave propagations in two and three dimensions and derive related ray tracing equations in a reduced phase space. The ray tracing equations can then be embedded into the level set framework to obtain an Eulerian method. In Section 4, we present the level set formulation for moving curves in three spatial dimensions that forms the basis of our algorithm. In Section 5, we show the numerical results of our algorithm on examples of anisotropic wave propagation in transversely isotropic media of hexagonal symmetry and media of orthorhombic symmetry to illustrate the advantages in accuracy and resolution of our approach. Finally, in Section 6, we conclude with a summary and a brief outline of future work.

\section{Geometric optics: background}

Throughout this paper, the Einstein summation convention is assumed for simplicity (see, e.g., [24]). Hooke's law states that the stress $\sigma_{i j}$ is related to the strain $e_{k l}$ through a stiffness tensor $C_{i j k l}$ and the relation

$$
\sigma_{i j}=C_{i j k l} e_{k l} \text {. }
$$

Therefore, the motion equation without body force takes the form

$$
\rho \frac{\partial^{2} \mathbf{U}}{\partial t^{2}}=\nabla \cdot \sigma
$$

where $\mathbf{U}=\left(U_{i}\right)$ is the displacement vector. By the relation between strain and displacement,

$$
e_{k l}=\frac{1}{2}\left(\frac{\partial U_{k}}{\partial x_{l}}+\frac{\partial U_{l}}{\partial x_{k}}\right)
$$

and the symmetry of the stiffness tensor, the motion equation is the wave equation

$$
\rho \frac{\partial^{2} U_{j}}{\partial t^{2}}=\frac{\partial}{\partial x_{i}}\left(C_{i j k l} \frac{\partial U_{k}}{\partial x_{l}}\right) .
$$

The geometric optics or high frequency approximation (see, e.g., [11,20]) assumes that the solution is of the form

$$
\mathbf{U}(x, t)=\sum_{n=0}^{\infty} \mathbf{A}^{(n)}(\mathbf{x}) f_{n}(t-\tau(\mathbf{x})), \quad f_{n+1}^{\prime}=f_{n},
$$

where the wavefront is given by

$$
t=\tau(\mathbf{x}) .
$$

Upon inserting the ansatz (4) into the wave equation and comparing individual coefficients of $f_{n-2}$ to zero successively, starting with the most singular term, $n=0$, we arrive at a recursive system for the phase $\tau$ and amplitudes $\mathbf{A}^{(n)}$. Because the geometric optics term consists of the phase and amplitude function determined by the zeroth order, $n=0$, and first order, $n=1$, term, respectively, we focus our attention here on the zeroth-order term.

Equating the zeroth-order term to zero gives rise to Christoffel's equation,

$$
\left(\Gamma_{j k}-\delta_{j k}\right) A_{k}^{(0)}=0
$$

which leads to the anisotropic eikonal equation for the phase function $\tau$,

$$
\operatorname{det}\left(a_{i j k l} p_{i} p_{l}-\delta_{j k}\right)=0,
$$

where $\mathbf{p}=\left(p_{i}\right)=\nabla \tau$ is the slowness vector normal to the wavefront (5), $\tau$ is the traveltime or phase of the wave mode, $a_{i j k l}=C_{i j k l} / \rho$ are the density-normalized elastic parameters, $\Gamma_{j k}$ equals $a_{i j k l} p_{i} p_{l}$, and $\delta_{j k}$ is the Kronecker 


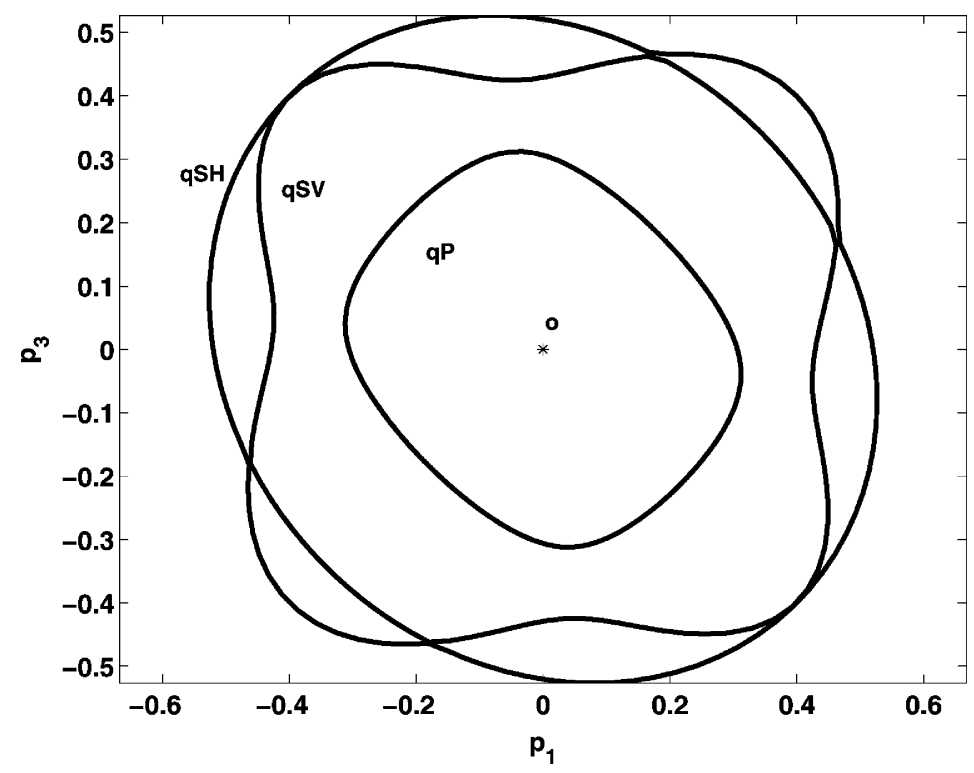

Fig. 1. The slowness surface for typical anisotropic media: a sextic surface which consists of three slowness sheets.

delta. Note that all of these quantities depend on the spatial coordinate vector $\mathbf{x}=\left(x_{1}, x_{2}, x_{3}\right)$, though we suppress this dependence in equations for notational simplicity.

Eq. (7) is a sextic polynomial equation in the slowness vector $\mathbf{p}$, i.e., the slowness vector $\mathbf{p}$ lies on a sextic surface consisting of three sheets, each surrounding the origin (see Fig. 1). To understand this, let

$$
p_{i}=\frac{n_{i}}{V}
$$

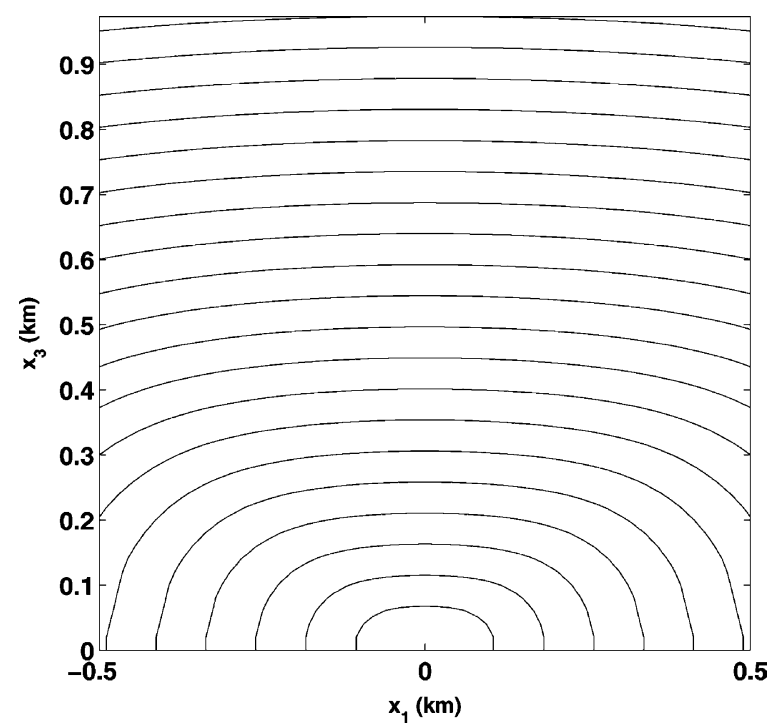

Fig. 2. The ray direction is not in general tangent to the wavefront normal in homogeneous anisotropic media, and thus the wavefronts are in general not spherical. The $\mathrm{qP}$ traveltime contours for zinc are also shown. 
where $\mathbf{n}=\left(n_{i}\right)$ is the unit normal vector to the wavefront and $V$ is the normal or phase speed of the wavefront. Then Eq. (7) yields

$$
\operatorname{det}\left(a_{i j k l} n_{i} n_{l}-V^{2} \delta_{j k}\right)=0,
$$

which is a cubic characteristic polynomial equation with respect to $V^{2}$ and hence has three eigenvalues corresponding to a quasi-longitudinal wave, known as the quasi-P wave, and two transverse waves, known as the quasi-SV and quasi-SH waves. Note we can extend $V$ to be homogeneous of degree 1 in the variable p. Moreover, experiments show that the velocity of the quasi-P wave is always greater than those of the transverse, or quasi-S, waves (see [18]). Hence the largest eigenvalue of Eq. (9) corresponds to quasi-P wave propagation, is simple, i.e., uniquely defined, and the quasi-P slowness sheet is the innermost one away from the other two. Furthermore, the quasi-P slowness sheet is convex (see, e.g., [24]), which is essential in constructing the paraxial approximation for the eikonal equations associated to the quasi-P waves [33].

In isotropic media, the polarization vector of the compression wave, known as the P-eigenvector, is tangent to the wavefront normal and the polarization vectors of the transverse waves, known as the SH- and SV-eigenvectors, are normal to the P-eigenvector, with the three eigenvectors forming an orthogonal system. But, as was shown by Musgrave [24], the P-eigenvector is in general not tangent to the wavefront normal in anisotropic media and hence the inclusion of the prefix "quasi” (see Fig. 2).

\section{The ray tracing system in a reduced phase space}

To illustrate the idea behind our approach, we first consider the two-dimensional anisotropic eikonal equation, i.e., Eq. (7) without the $p_{3}$ component. Consequently, we denote the resultant slowness surface in the two-dimensional case by

$$
F\left(x_{1}, x_{2}, p_{1}, p_{2}\right)=0,
$$

and parametrize the slowness vector by

$$
p_{1}=\frac{\cos \theta}{V\left(x_{1}, x_{2}, \theta\right)}, \quad p_{2}=\frac{\sin \theta}{V\left(x_{1}, x_{2}, \theta\right)},
$$

where $\theta$ is known as the phase angle, varying from $-\pi$ to $\pi$, and $V$ the phase velocity solving an eigenvalue problem. Applying the method of characteristics (see, e.g., [11]) to Eq. (10) yields

$$
\begin{aligned}
\frac{\mathrm{d} x_{1}}{\mathrm{~d} t} & =\left(p_{1} \frac{\partial F}{\partial p_{1}}+p_{2} \frac{\partial F}{\partial p_{2}}\right)^{-1} \frac{\partial F}{\partial p_{1}}, \\
\frac{\mathrm{d} x_{2}}{\mathrm{~d} t} & =\left(p_{1} \frac{\partial F}{\partial p_{1}}+p_{2} \frac{\partial F}{\partial p_{2}}\right)^{-1} \frac{\partial F}{\partial p_{2}}, \\
\frac{\mathrm{d} p_{1}}{\mathrm{~d} t} & =-\left(p_{1} \frac{\partial F}{\partial p_{1}}+p_{2} \frac{\partial F}{\partial p_{2}}\right)^{-1} \frac{\partial F}{\partial x_{1}}, \\
\frac{\mathrm{d} p_{2}}{\mathrm{~d} t} & =-\left(p_{1} \frac{\partial F}{\partial p_{1}}+p_{2} \frac{\partial F}{\partial p_{2}}\right)^{-1} \frac{\partial F}{\partial x_{2}}
\end{aligned}
$$

where the evolution parameter $t$ has the dimension of time. Here since the wavefront is expanding, time can be used as an evolution parameter along the ray; the above ray tracing system is obtained with this observation. To obtain an equation for $\mathrm{d} \theta / \mathrm{d} t$, we differentiate the equations in (11), arriving at

$$
\frac{\mathrm{d} p_{1}}{\mathrm{~d} t}=\frac{-V \sin \theta-(\partial V / \partial \theta) \cos \theta}{V^{2}} \frac{\mathrm{d} \theta}{\mathrm{d} t}-\frac{\cos \theta}{V^{2}}\left(\frac{\partial V}{\partial x_{1}} \frac{\mathrm{d} x_{1}}{\mathrm{~d} t}+\frac{\partial V}{\partial x_{2}} \frac{\mathrm{d} x_{2}}{\mathrm{~d} t}\right),
$$




$$
\frac{\mathrm{d} p_{2}}{\mathrm{~d} t}=\frac{V \cos \theta-(\partial V / \partial \theta) \sin \theta}{V^{2}} \frac{\mathrm{d} \theta}{\mathrm{d} t}-\frac{\sin \theta}{V^{2}}\left(\frac{\partial V}{\partial x_{1}} \frac{\mathrm{d} x_{1}}{\mathrm{~d} t}+\frac{\partial V}{\partial x_{2}} \frac{\mathrm{d} x_{2}}{\mathrm{~d} t}\right) .
$$

Thus, solving the above equations for $\mathrm{d} \theta / \mathrm{d} t$ and substituting in (14) and (15) leads to

$$
\frac{\mathrm{d} \theta}{\mathrm{d} t}=\left(p_{1} \frac{\partial F}{\partial p_{1}}+p_{2} \frac{\partial F}{\partial p_{2}}\right)^{-1}\left(V \frac{\partial F}{\partial x_{1}} \sin \theta-V \frac{\partial F}{\partial x_{2}} \cos \theta\right) .
$$

Eqs. (12), (13) and (18), in total, give us the velocity field needed in our level set formulation for two-dimensional anisotropic wave propagation. Similarly, the ray tracing system for the three-dimensional case gives the desired velocity field in that case.

\section{Level set formulation for moving curves in reduced phase space}

We now place the problem into the level set framework found in [7], with the process similar to that in [26]. This is desirable for numerical reasons, with the advantages in automatic resolution and the ability to easily and accurately handle multi-valued wavefronts. We first begin with the setup introduced in [17]. In the two-dimensional ray tracing problem, the objects of interest we will evolve, in place of the wavefronts, form curves in three-dimensional reduced phase space. This space, for fixed time, consists of the points $\left(x_{1}, x_{2}, \theta\right)$, where $\mathbf{x}=\left(x_{1}, x_{2}\right) \in \mathbf{R}^{2}$, representing points in spatial space, and $\theta \in[-\pi, \pi]$, representing the phase angle. The link is that the projection of these curves to spatial space gives back the wavefronts we wish to construct. The advantage to working in reduced phase space is that the curves there are smooth, even when their projections to spatial space, the wavefronts, are multi-valued. This is what allows us to effectively handle multi-valued wavefronts.

The level set framework for the ray tracing consists of implicitly representing the curves of interest in reduced phase space. This is through the use of two real valued level set functions, $\phi$ and $\psi$, over reduced phase space and time whose common zeros, $\phi=\psi=0$, for a fixed time give the curves at that moment. In fact, $\phi$ and $\psi$ can be thought of as the components of a two component vector valued level set function. Note reduced phase space can be replaced by $\mathbf{R}^{3}$ through enforcing periodicity in the $\theta$ direction. The evolution of wavefronts thus translates to that of curves in $\mathbf{R}^{3}$ which furthermore, in our framework, translates to evolution of the level set functions. We achieve this through a system of PDEs for $\phi$ and $\psi$ incorporating the velocity for the motion of the curves. As seen from Eqs. (12), (13) and (18), for a given slowness surface defined by $F=0$ and phase velocity $V$ corresponding to the $\mathrm{qP}, \mathrm{qSV}$, or qSH waves, the points on the curves move with velocity

$$
\mathbf{w}\left(x_{1}, x_{2}, \theta\right)=\left(\begin{array}{c}
\left(p_{1} \frac{\partial F}{\partial p_{1}}+p_{2} \frac{\partial F}{\partial p_{2}}\right)^{-1} \frac{\partial F}{\partial p_{1}} \\
\left(p_{1} \frac{\partial F}{\partial p_{1}}+p_{2} \frac{\partial F}{\partial p_{2}}\right)^{-1} \frac{\partial F}{\partial p_{2}} \\
\left(p_{1} \frac{\partial F}{\partial p_{1}}+p_{2} \frac{\partial F}{\partial p_{2}}\right)^{-1}\left(V \frac{\partial F}{\partial x_{1}} \sin \theta-V \frac{\partial F}{\partial x_{2}} \cos \theta\right)
\end{array}\right),
$$

in reduced phase space, noting that $p_{1}$ and $p_{2}$ can be written in terms of $\theta$ and $V$. The velocity can thus be defined in all of reduced phase space and used to evolve the level set functions $\phi$ and $\psi$ also defined there. Note also it is fixed in time and for any given curve and, furthermore, it is a generalization of the velocity field considered in [26]. In fact, the algorithm we construct here is the same as that in [26] except for the use of this more general form of the velocity for the anisotropic case. The evolution of the level set functions under this velocity satisfies the system of PDEs

$$
\phi_{t}+\mathbf{w} \cdot \nabla \phi=0, \quad \psi_{t}+\mathbf{w} \cdot \nabla \psi=0,
$$


which is a system of transport equations. Thus, to find the position of wavefronts at a given time, the procedure for our algorithm is to solve the evolution equations for the level set functions in $\mathbf{R}^{3}$, then project the common points of the zero level sets to spatial space. Note, the two evolution equations can even be solved separately since they are not coupled.

The numerical aspects of this algorithm include placing an Eulerian fixed grid in $\mathbf{R}^{3}$ as a foundation for solving the evolution PDEs. This provides the automatic resolution of wavefronts we desire. It also allows for easy discretization of PDEs through the use of standard and well-studied techniques. In this case, the PDEs we are interested in are of Hamilton-Jacobi form and can be discretized using, for example, fourth-order SSP-RK [40], a recently developed Runge-Kutta scheme, in time and fifth-order WENO-Godunov [19] in space. Thus, implementing such a discretization and iterating up to the desired time will give the curves of interest in $\mathbf{R}^{3}$, regardless of whether the wavefronts have become multi-valued in the process. As seen in [26], one correction, in general, has to be made to this algorithm. The zero level set surfaces of the level set functions we are evolving may develop kinks or become nearly parallel to each other at certain points. This can greatly affect the accuracy of the discretization as well as the plotter, which is based on interpolation. To prevent this from occurring, reinitialization can be performed every few time steps. This involves replacing the level set functions with level set functions of a better form by evolving them under PDEs that use distance and orthogonality as criteria. For more details, see [26]. Note one effect of incorporating reinitialization is that the evolution of $\phi$ and $\psi$ become coupled. Also, we note that in simulations, $\theta$ is sometimes scaled to lie in $[-1,1]$ through $\theta \mapsto \theta / \pi$.

This altogether gives the framework and numerical discretization we use to construct wavefronts and determine traveltimes. Efficient and optimal algorithms for the implementation following local level set ideas [1,29] are being pursued to achieve faster speeds and for larger problems. Such algorithms will be needed to handle the case of ray tracing in three dimensions, where the basic formulation under our approach is a straightforward extension of that in the two-dimensional case. See [26] for details.

\section{Numerical results}

Although a general anisotropic solid has 21 independent elastic parameters, the transversely isotropic, or TI, solid has only five. It nevertheless retains the essential features of the anisotropic case that we are interested in. Therefore, it is convenient to use TI solids as models to illustrate the advantages of our approach. We first consider the simplest case for TI solids, those with vertical symmetry axes, known as VTI solids. We then test our approach on inclined TI models obtained by rotating the VTI models [31]. Because the slowness surface equation for the inclined TI model is a sextic polynomial equation with the essential features of a general sextic slowness surface and admits no explicit solutions, it is suitable for us to use this model as a further test of our proposed algorithm. The extensive numerical experiments we present show that our algorithm is accurate, even in situations with multi-valued solutions.

The elastic modulus matrix for transversely isotropic media with vertical symmetry axes has five independent components among a total of twelve nonzero components (see, e.g., [24]). A closed form solution exists in this case for the eigenvalue problem (7). The quasi-P and quasi-SV slowness surfaces for VTI can be represented by a quartic polynomial equation and the quasi-SH slowness surface can be decoupled from this, leading to the equations

$$
c_{1} p_{1}^{4}+c_{2} p_{1}^{2} p_{2}^{2}+c_{3} p_{2}^{4}+c_{4} p_{1}^{2}+c_{5} p_{2}^{2}+1=0,
$$

and

$$
\frac{1}{2}\left(a_{11}-a_{12}\right) p_{1}^{2}+a_{44} p_{2}^{2}=1,
$$

where

$$
\begin{aligned}
& c_{1} \equiv a_{11} a_{44}, \quad c_{2} \equiv a_{11} a_{33}+a_{44}^{2}-\left(a_{13}+a_{44}\right)^{2}, \quad c_{3} \equiv a_{33} a_{44}, \\
& c_{4} \equiv-\left(a_{11}+a_{44}\right), \quad c_{5} \equiv-\left(a_{33}+a_{44}\right) .
\end{aligned}
$$

In the above equations, the Voigt recipe is assumed to simplify the elasticity tensor $a_{i j k l}$ to obtain $a_{i j}$. 

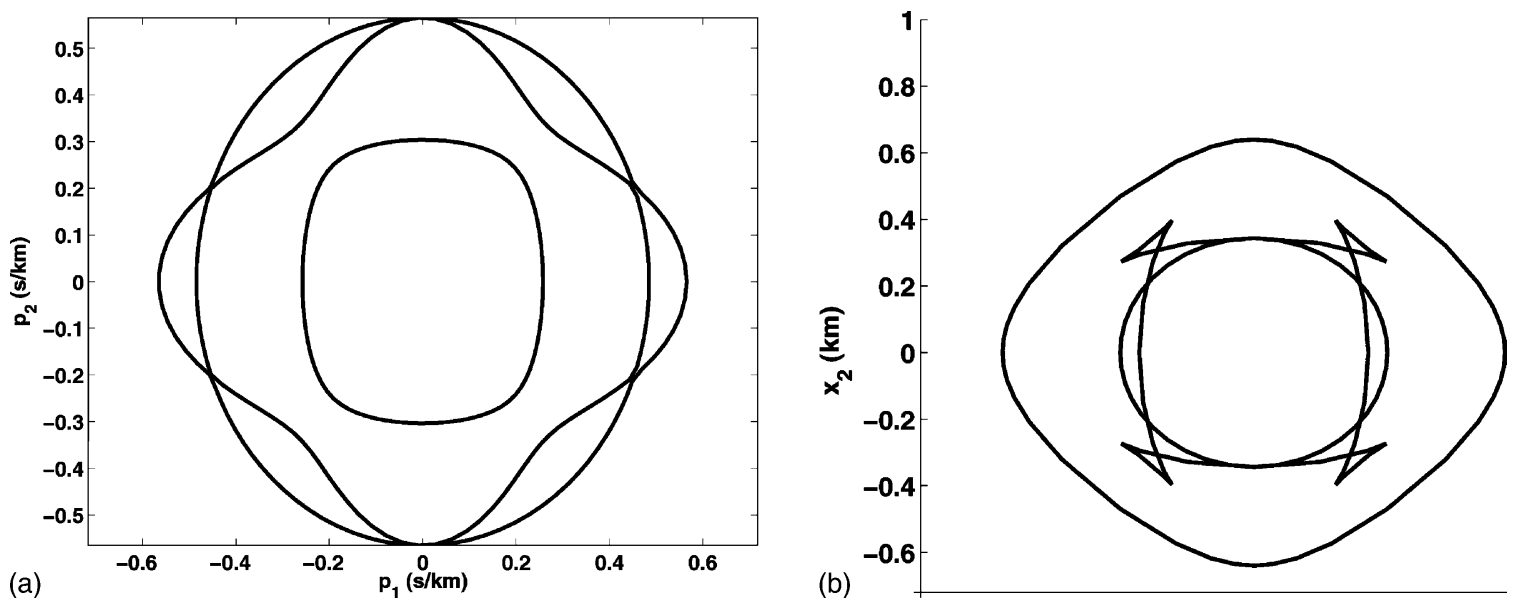

Fig. 3. Reciprocity between slowness surfaces and wavefronts for Greenriver shale: (a) slowness surfaces; (b) wavefronts.

Thus, the phase velocities for the three different waves take the form

$$
\begin{aligned}
& V_{\mathrm{qP}}^{2}=\frac{1}{2}\left(-Y_{1}+\sqrt{Y_{1}^{2}-4 Y_{2}}\right), \quad V_{\mathrm{qSV}}^{2}=\frac{1}{2}\left(-Y_{1}-\sqrt{Y_{1}^{2}-4 Y_{2}}\right), \\
& V_{\mathrm{SH}}^{2}=\frac{1}{2}\left(a_{11}-a_{12}\right) \cos ^{2} \theta+a_{44} \sin ^{2} \theta,
\end{aligned}
$$

where

$$
Y_{1}=c_{4} \cos ^{2} \theta+c_{5} \sin ^{2} \theta, \quad Y_{2}=c_{1} \cos ^{4} \theta+c_{2} \cos ^{2} \theta \sin ^{2} \theta+c_{3} \sin ^{4} \theta .
$$

These velocities are obtained by substituting (11) into (19) and (20) and solving the resulting polynomial equations. We can also obtain phase velocities associated to inclined TI media [31] in a similar manner.

In the following examples, we simulate wave propagations excited by point sources, perhaps modelled by delta functions. This, in turn, implies that the initial level set functions can be easily determined.

As a first example, we compute the three waves for Greenriver shale, a typical VTI medium [46]. The five elastic parameters are $a_{11}=15.0638, a_{33}=10.8373, a_{13}=1.6381, a_{44}=3.1258$, and $a_{12}=6.5616$. Fig. 3(a) shows the three slowness surfaces of the different waves. The innermost one corresponds to the $\mathrm{qP}$ wave, the one with dimples to the qSV wave, and the outer elliptical surface to the qSH wave (see Eq. (20)). In slowness space, the three slowness surfaces for the $\mathrm{qP}, \mathrm{qSV}$, and $\mathrm{qSH}$ waves are from innermost to outermost, however, because of the reciprocity between the slowness space and position space, the wavefronts for these same waves are from outermost to innermost in position space, as shown in Fig. 3(b). Fig. 3(b) shows the three wavefronts for the waves at the moment $t=0.1942 \mathrm{~s}$. Because the $\mathrm{qP}$ wave is the fastest among the three, the outermost wavefront corresponds to $\mathrm{qP}$ wave propagation. Note the cusps accompanying the qSV wave are well-captured. The innermost ellipse is the qSH wavefront because of the elliptical anisotropy characterized by Eq. (20). Fig. 4(a) shows a calibration result for the $\mathrm{qP}$ wavefront. The darker curve segment imposed on the outermost wavefront represents the same wavefront computed using a paraxial eikonal solver for $\mathrm{qP}$ waves [31]. Note the two $\mathrm{qP}$ wavefront segments match perfectly. Calibration, used here, means comparing our computational result with those obtained by other alternative approaches; by doing this we can verify that our computational results are correct. The paraxial eikonal solver in $[31,33,34]$ is developed specifically for capturing qP wavefronts. Furthermore, its theory is developed in [35] and can be generalized to treat general Hamilton-Jacobi equations with convex Hamiltonians. Fig. 4(b) shows a calibration result for the qSV wavefront alternatively calculated using a ray tracing method. Note the results match for this case of multi-valued wavefronts as well. The ray tracing method used here involves solving an ODE system similar to 

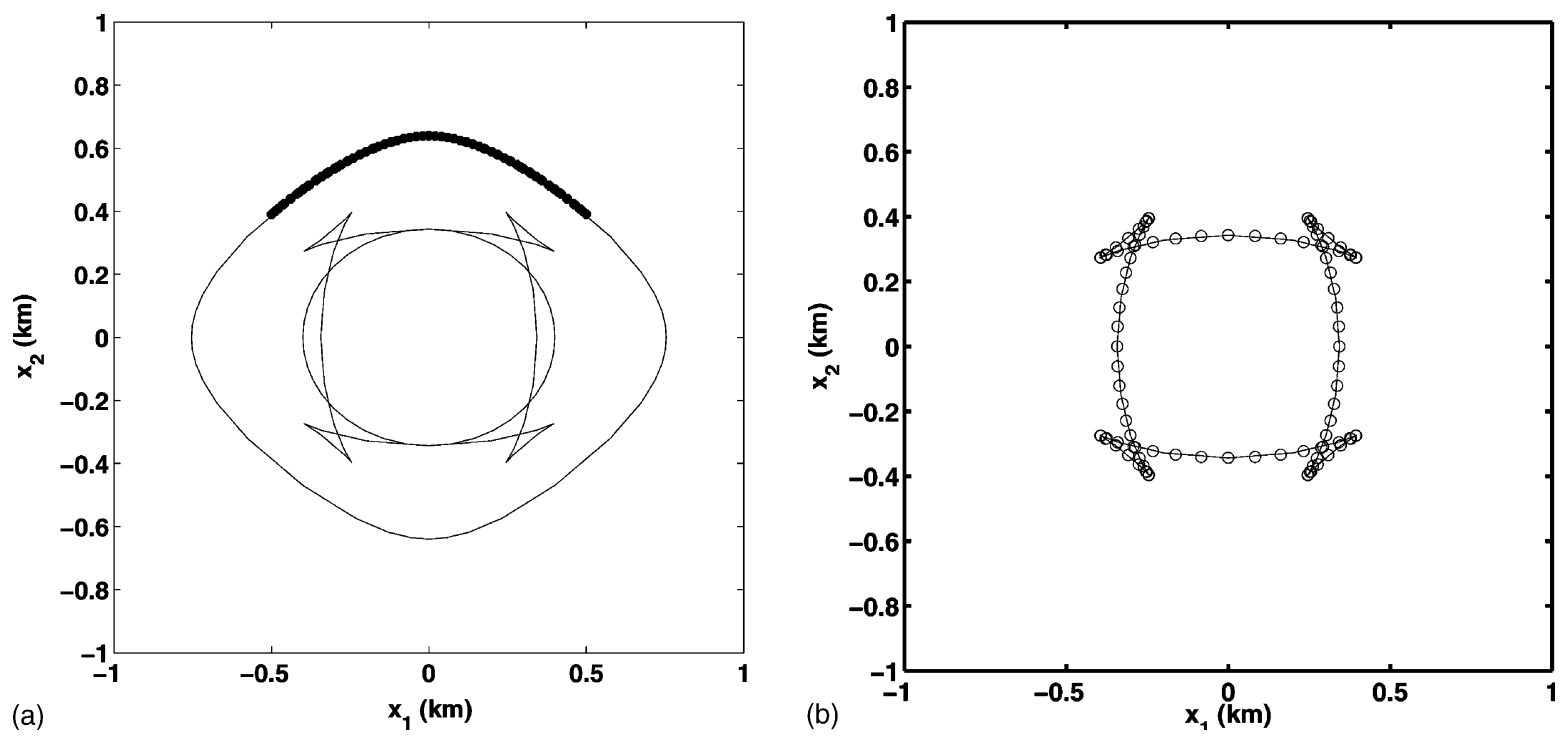

Fig. 4. Calibration for wavefronts: (a) a segment of quasi-P wavefront computed by a paraxial eikonal solver is imposed on the quasi-P wavefront computed using our method; (b) a qSV wavefront computed using a ray tracing method, shown in circles, is imposed on the wavefront computed from our method, shown as a solid curve.

(12)-(15) by a Matlab ODE45 solver. Fig. 5 shows projections of the three waves on the $x_{2} \theta$-plane. All three curves are single-valued functions of the phase angle $\theta$, which agrees with classical results in Maslov theory [4].

Since VTI media have rotational invariance about the $x_{2}$-axis, revolving a computed wavefront in two dimensions about the $x_{2}$-axis yields the desired wavefront in three dimensions. Figs. 6-10 show three-dimensional examples
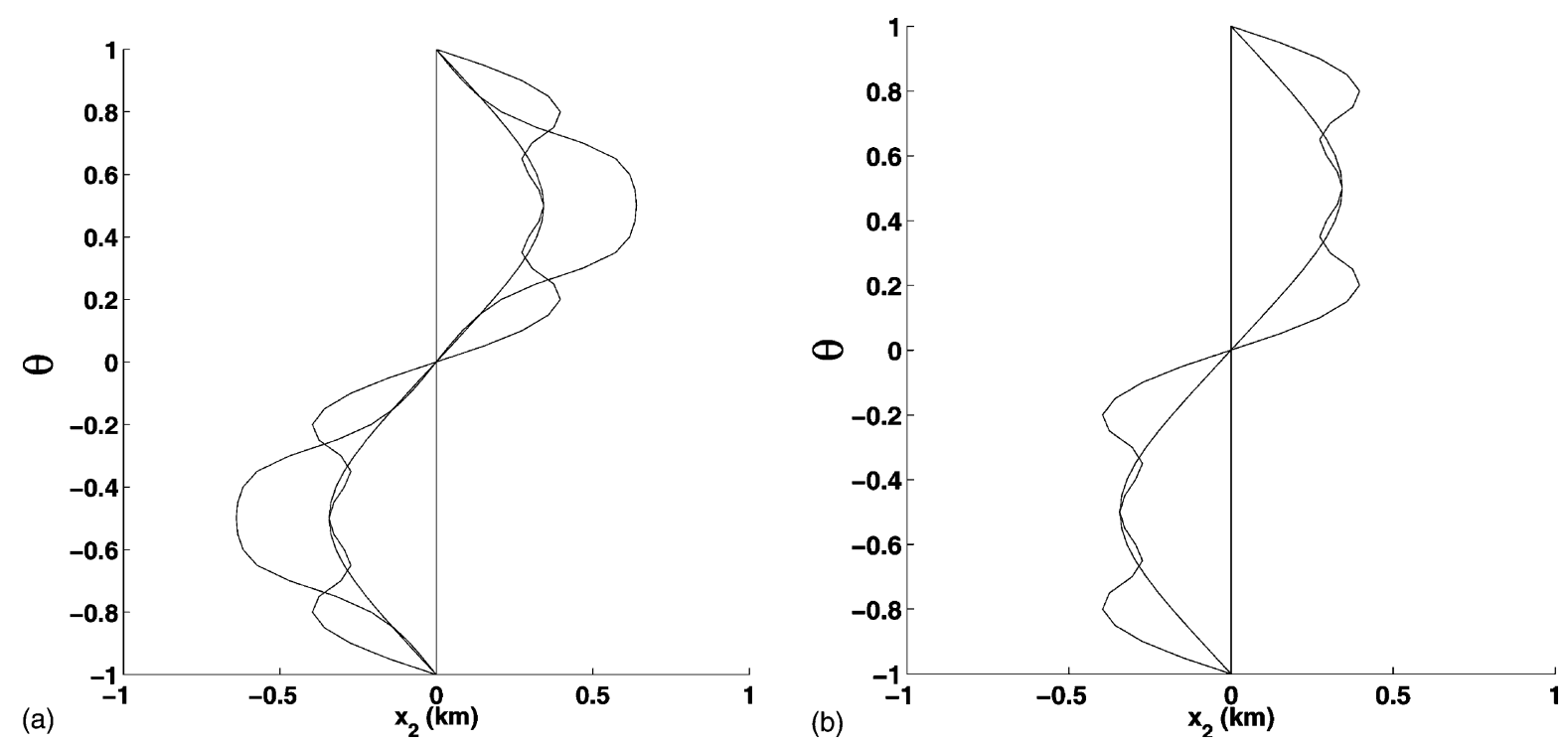

Fig. 5. Perspective of the function in three dimensions on the $x_{2} \theta$-plane: the three curves corresponding to three waves are single-valued functions of the phase angle $\theta$. Shown are the (a) qP, qSV and qSH waves; (b) qSV and qSH waves. 


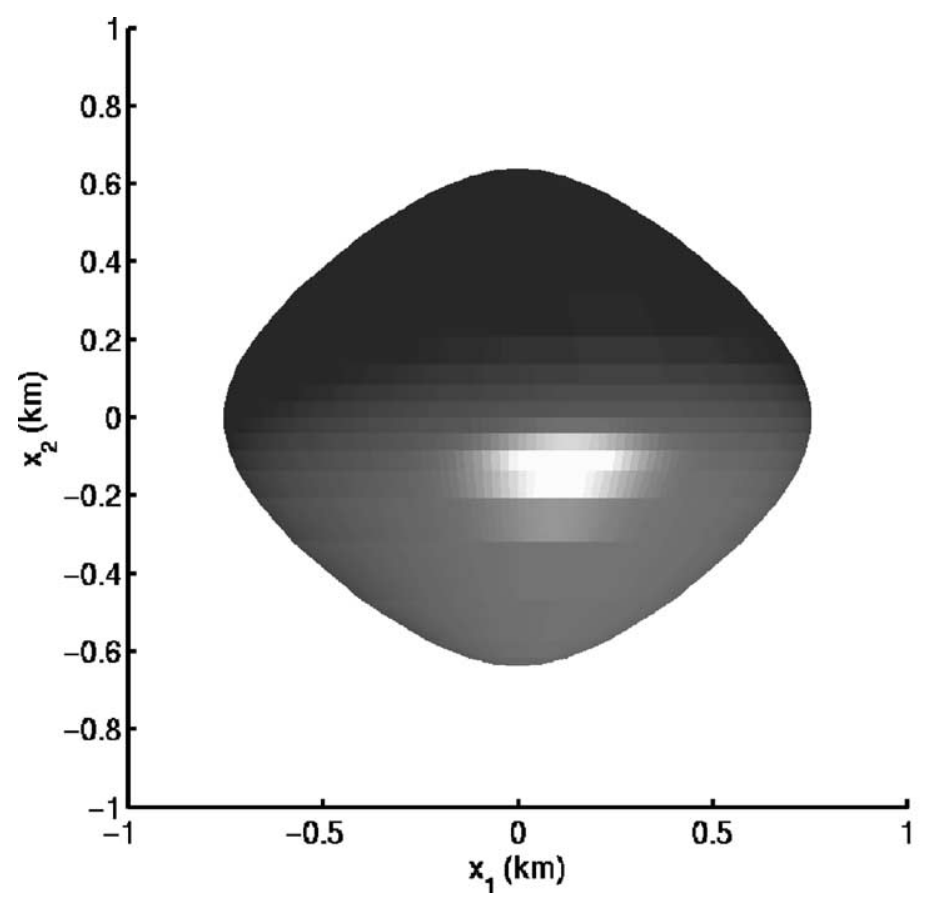

Fig. 6. A qP wavefront in three dimensions obtained by rotating the corresponding qP wavefront in two dimensions: Greenriver shale.

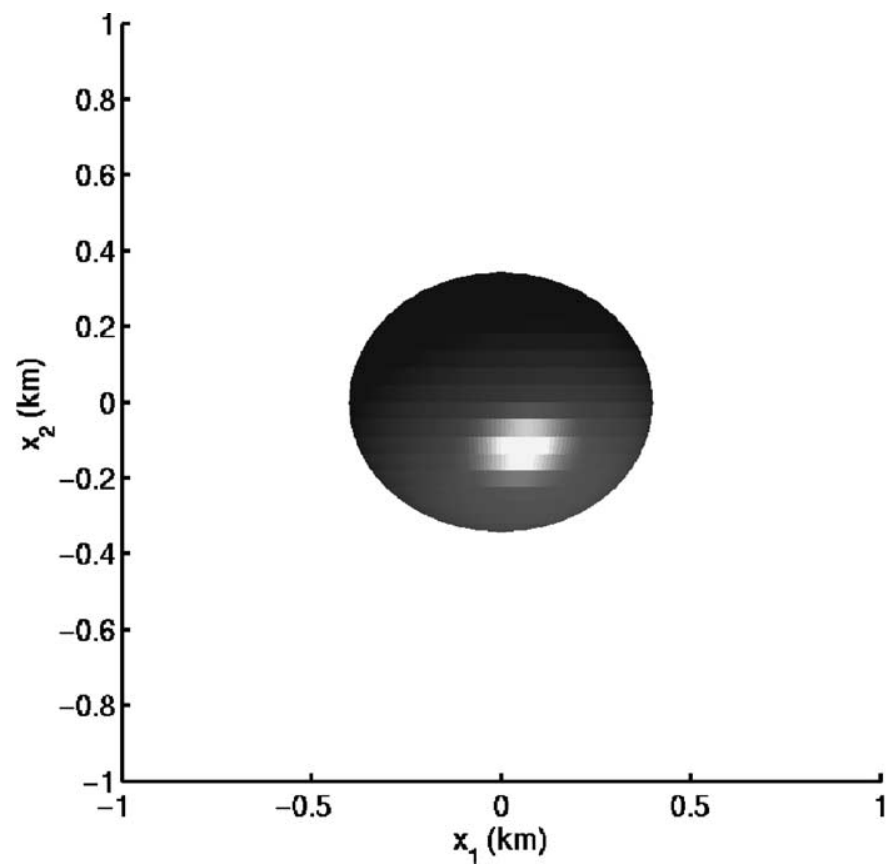

Fig. 7. A qSH wavefront in three dimensions obtained by rotating the corresponding qSH wavefront in two dimensions: Greenriver shale. 


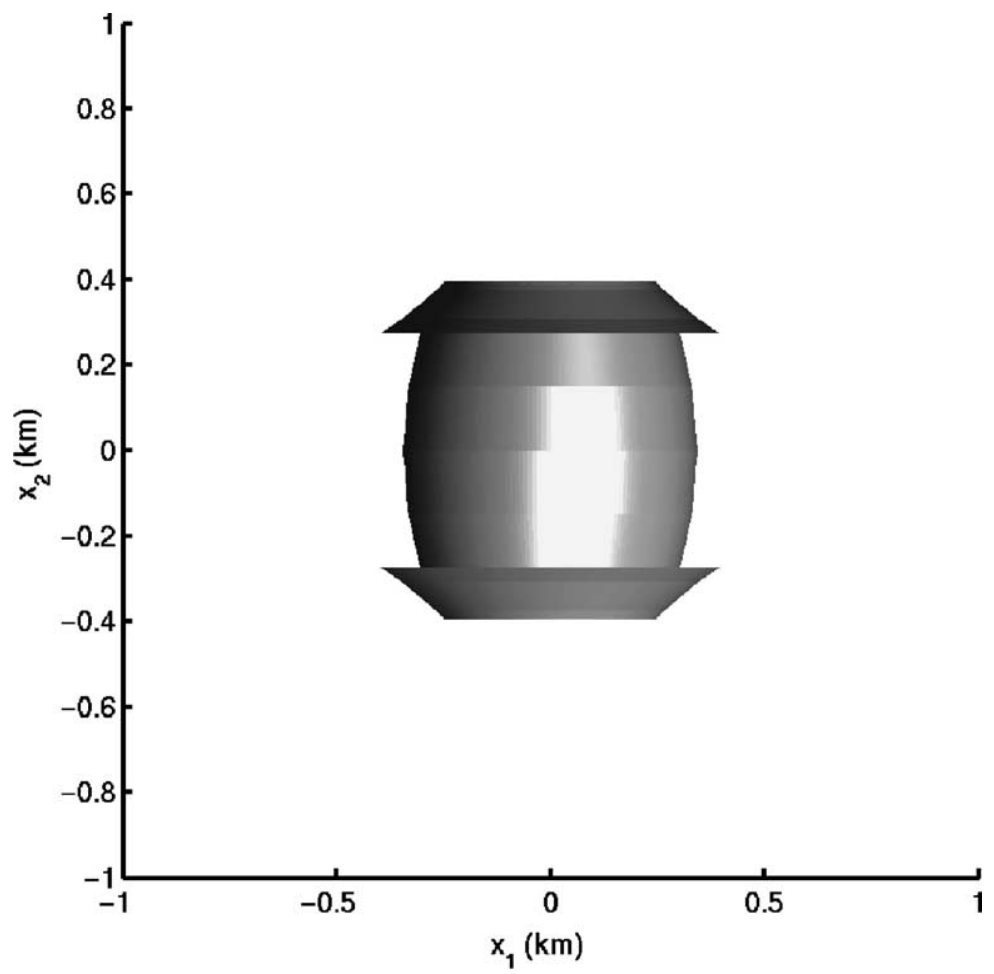

Fig. 8. A qSV wavefront in three dimensions obtained by rotating the corresponding qSV wavefront in two dimensions for Greenriver shale: a horizontal perspective of the wavefront.

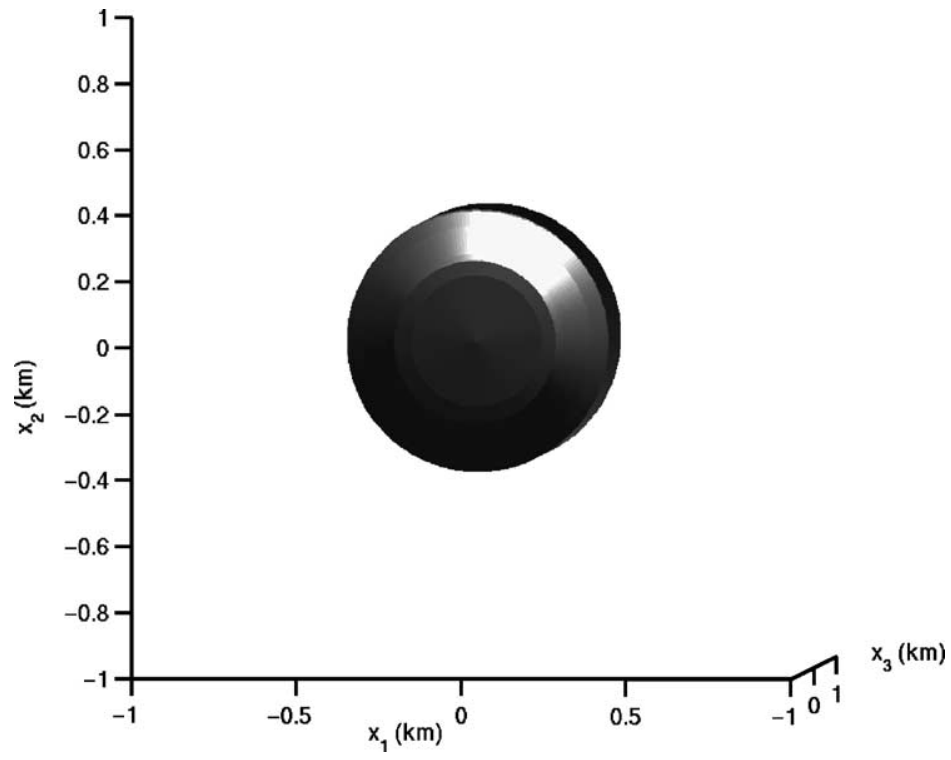

Fig. 9. A qSV wavefront in three dimensions obtained by rotating the corresponding qSV wavefront in two dimensions for Greenriver shale: the horizontal sections are circles because of the transverse isotropy of VTI media. 


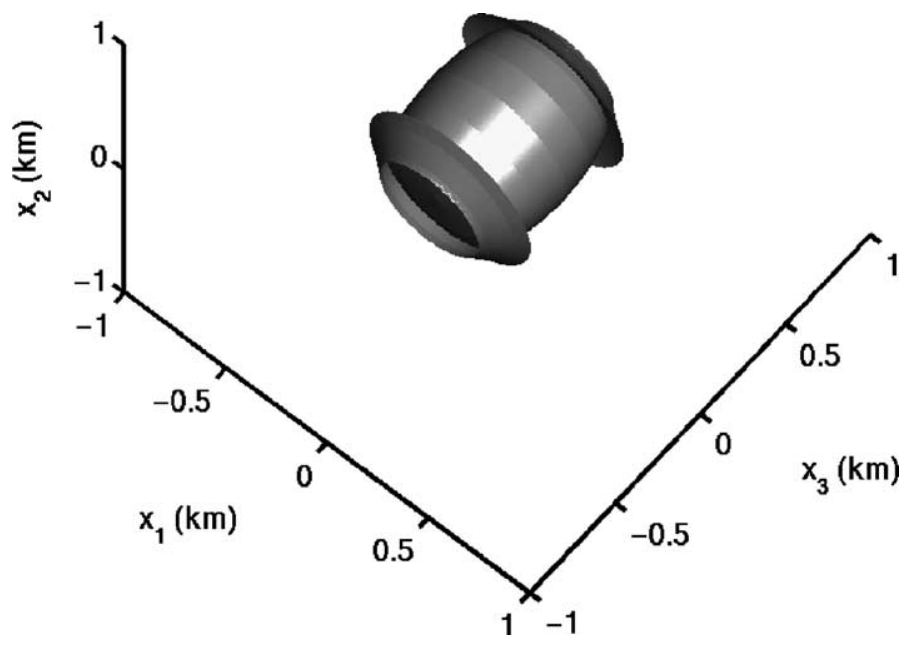

Fig. 10. A qSV wavefront in three dimensions obtained by rotating the corresponding qSV wavefront in two dimensions for Greenriver shale: the cuspidal edges are clearly seen.

of wavefronts after rotation of the computations of Greenriver shale in Fig. 3(b). We plot the wavefronts separately for illustrational purposes as they are nested. Figs. 6 and 7 show the qP and qSH wavefronts, respectively, and Figs. 8-10 show different views of the more interesting qSV wavefront. In these pictures, Fig. 8 has a horizontal perspective, where the profile can be compared to the qSV wavefront in two dimensions, Fig. 9 illustrates that the horizontal sections of the qSV wavefront are circles due to the transverse isotropy of the VTI medium, and Fig. 10 shows the cuspidal edges from a different point of view.

To test the resolution of our approach in capturing cusps, we compute the qSV wavefronts for Beryl, a VTI crystal. Fig. 11(a) shows our results of an expanding qSV wavefront at three different times. Note there are four
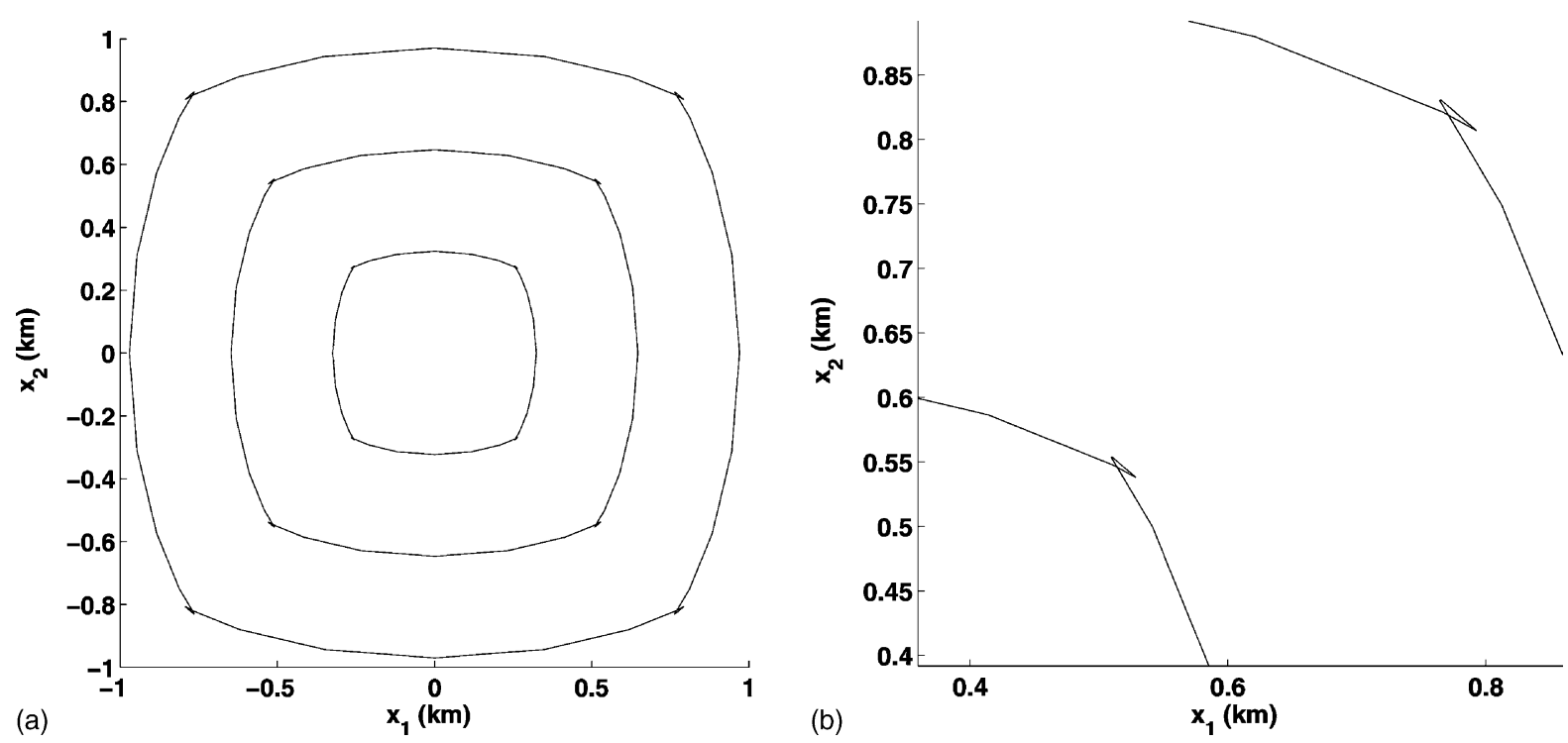

Fig. 11. Resolution test on Beryl: (a) four tiny cusps accompanying the qS waves; (b) the cusps at the upper right corner are zoomed in. 

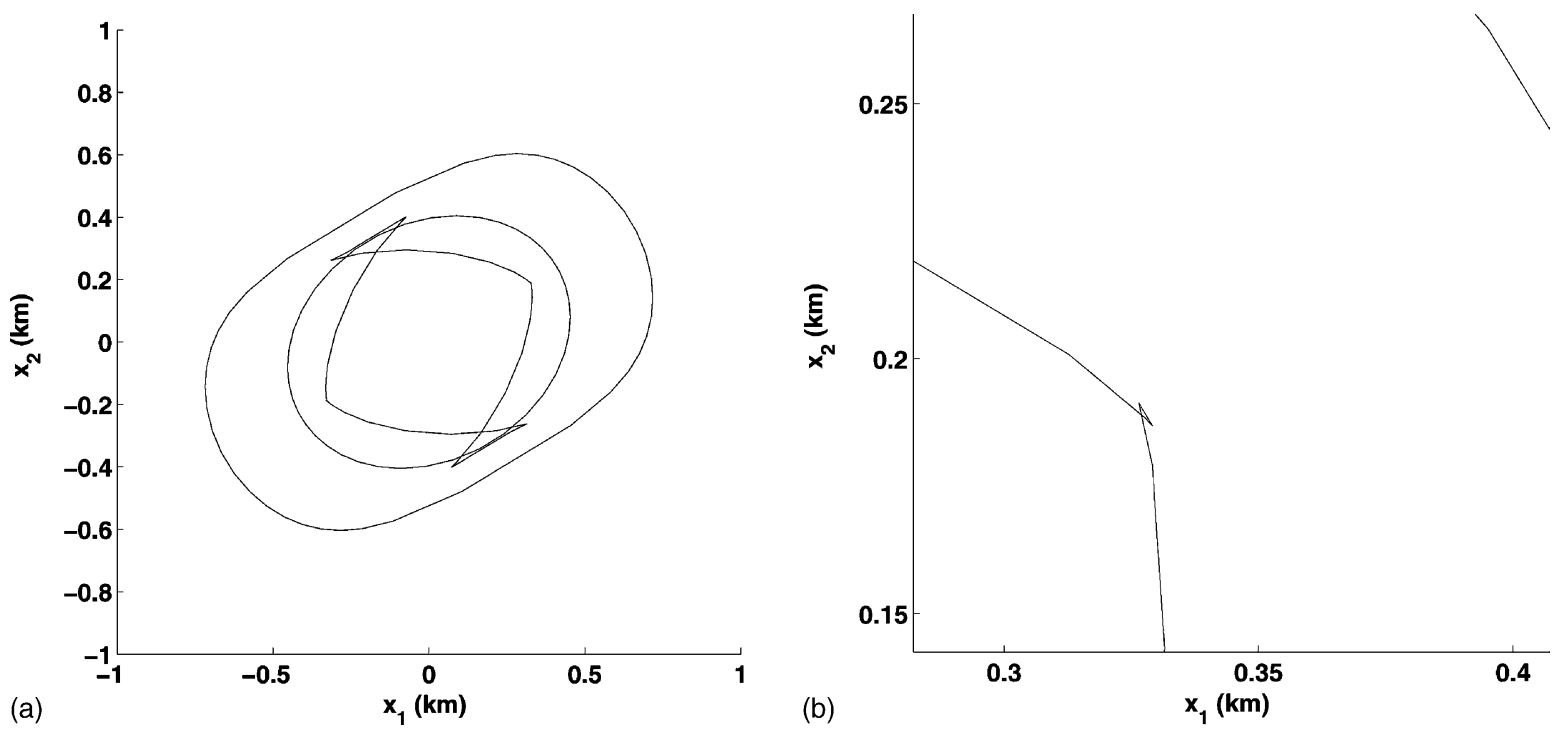

Fig. 12. Wavefronts for an inclined zinc model: (a) two cusps accompanying the qSV wave are clearly seen; (b) another cusp of the qSV wave at upper right corner is zoomed in.

tiny cusps in the wavefront at each time because of the instantaneous singularity. Fig. 11(b) shows an enlargement of the upper right cusps of the two outer wavefronts. Here, an Eulerian mesh of size $50 \times 50 \times 50$ is placed over the computational domain, $[-1,1] \times[-1,1] \times[-1,1]$. This shows that the cusps are still well-captured under a coarse mesh.

For inclined TI media, we compute the wavefronts for the zinc model rotated by $30^{\circ}$. Fig. 12 (a) shows the three wavefronts at the moment $t=0.1891 \mathrm{~s}$. The one corresponding to the qSV wavefront has four cusps, with two of

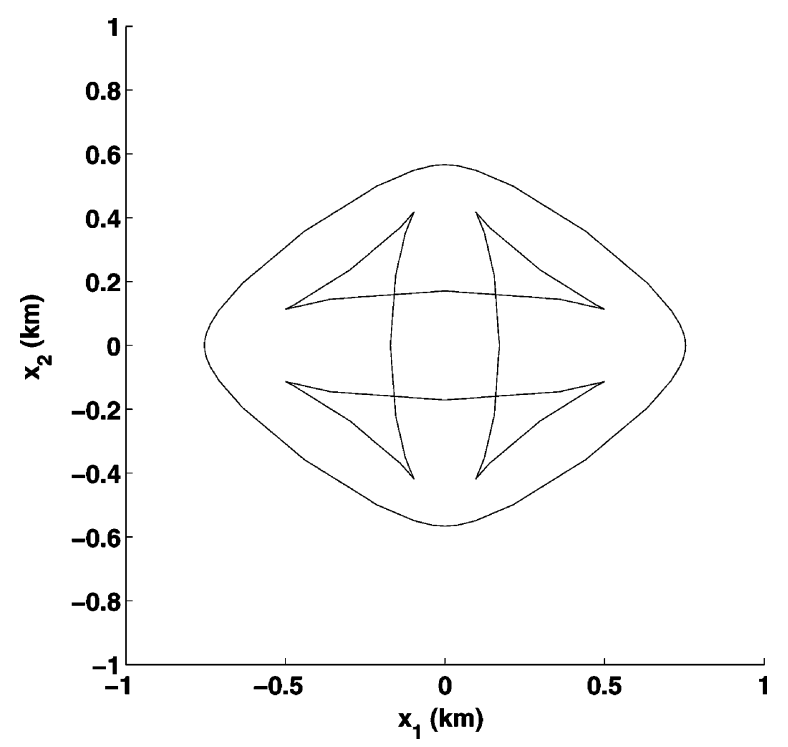

Fig. 13. Wavefronts for an orthorhombic medium: spruce, a soft wood of anomalous properties. The qP and qSV wavefront with cusps are shown. 
them apparent in Fig. 12(a). One of the other two can be seen in the enlargement in Fig. 12(b). Since the slowness surface in an inclined TI medium satisfies a general sextic polynomial equation, this is a strong indication that our proposed approach can handle arbitrary two-dimensional anisotropic media.

To test our approach on capturing qSV cusps for general anisotropic media, we compute the qSV wavefronts for spruce, which has an orthorhombic symmetry [24]. Although in this case, the two-dimensional slowness surface equation is still a sextic polynomial equation, rotational invariance about the $x_{2}$-axis no longer holds, with the cusps of qSV waves only appearing on a particular plane [24]. Furthermore, spruce is highly anisotropic. Fig. 13 shows the $\mathrm{qP}$ and $\mathrm{qSV}$ wavefronts of spruce at a certain time.

\section{Conclusion}

We have created and tested a new Eulerian method for solving anisotropic eikonal equations that is able to capture and resolve all three wave modes inherent in the wave propagations. This especially includes the qSV waves, a class of multi-valued solutions, which may develop cusps. The method is based on a level set formulation for moving curves in three dimensions and is seen for the isotropic case in [26]. The level set framework allows it to automatically handle and resolve multi-valued solutions, should they appear, with numerical results clearly demonstrating this. We expect our new method to find its way into anisotropic seismic imaging tool boxes, anisotropic velocity analysis, and numerous other areas and applications.

In future work, we plan to compute amplitudes for the three wave modes under the same Eulerian framework so that a complete geometric optics term can be constructed for high resolution seismic imaging. See [34] for an Eulerian approach to constructing a complete geometric optics term for quasi-P waves in the framework of paraxial geometric optics. Furthermore, we plan to improve the efficiency of the method by localizing the level set evolution around the zero level set to obtain a $\mathrm{O}(N \log N)$ algorithm, where $N$ is the number of points in the grid. The work in [29] can be consulted in this direction. Finally, the framework of our current approach can be easily extended to the three-dimensional case, which is a project we are currently pursuing. The results of these will be reported elsewhere.

\section{Acknowledgements}

J. Qian would like to thank Professors B. Cockburn, F. Santosa, and W.W. Symes for their interest and encouragement in this work. Li-Tien Cheng was supported by NSF Grant \#0112413 and \#0208449 and Stanley Osher was supported by AFOSR Grant \#F49620-01-1-0189.

\section{References}

[1] D. Adalsteinsson, J.A. Sethian, A fast level set method for propagating interfaces, J. Comput. Phys. 118 (1995) 269-277.

[2] J.-D. Benamou, Big ray tracing: multivalued travel time field computation using viscosity solutions of the eikonal equations, J. Comput. Phys. 128 (1996) 463-474.

[3] J.-D. Benamou, Direct solution of multi-valued phase-space solutions for Hamilton-Jacobi equations, Commun. Pure Appl. Math. 52 (1999) 1443-1475.

[4] J.-D. Benamou, I. Solliec, A Eulerian method for capturing caustics, J. Comput. Phys. 162 (2000) 132-163.

[5] G. Beylkin, Imaging of discontinuities in the inverse scattering problem by inversion of a causal generalized radon transform, J. Math. Phys. 26 (1985) 99-108.

[6] G. Beylkin, R. Burridge, Linearized inverse scattering problem of acoustics and elasticity, Wave Motion 12 (1990) 15-22.

[7] P. Burchard, L.-T. Cheng, B. Merriman, S. Osher, Motion of curves in three spatial dimensions using a level set approach, J. Comput. Phys. 170 (2) (2001) 720-741.

[8] R. Burridge, M.V. de Hoop, D. Miller, C. Spencer, Multiparameter inversion in anisotropic media, Geophys. J. Int. 134 (1998) $757-777$.

[9] V. Cerveny, Seismic rays and ray intensities in inhomogeneous anisotropic media, Geophys. J. R. Astr. Soc. 29 (1972) 1-13. 
[10] V. Cerveny, I.A. Molotkov, I. Psencik, Ray Method in Seismology, Univerzita Karlova Press, Praha, 1977.

[11] R. Courant, D. Hilbert, Methods of Mathematical Physics, vol. II, Wiley, New York, 1962.

[12] M.V. de Hoop, N. Bleistein, Generalized radon transform inversions for reflectivity in anisotropic elastic media, Inverse Problems 13 (1997) 669-690.

[13] M.V. de Hoop, C. Spencer, R. Burridge, The resolving power of seismic amplitude data: an anisotropic inversion/migration approach, Geophysics 64 (1999) 852-873.

[14] W. Debski, A. Tarantola, Information on elastic parameters obtained from the amplitudes of reflected waves, Geophysics 60 (1995) $1426-$ 1436.

[15] B. Engquist, E. Fatemi, S. Osher, Numerical resolution of the high frequency asymptotic expansion of the scalar wave equation, J. Comput. Phys. 120 (1995) 145-155.

[16] B. Engquist, O. Runborg, Multi-phase computations in geometrical optics, J. Comput. Appl. Math. 74 (1996) 175-192.

[17] B. Engquist, O. Runborg, A.-K. Tornberg, High frequency wave propagation by the segment projection method, J. Comput. Phys. 178 (2002) 373-390

[18] F.I. Fedorov, Theory of Elastic Waves in Crystals, Plenum Press, New York, 1968.

[19] G.S. Jiang, D. Peng, Weighted ENO schemes for Hamilton-Jacobi equations, SIAM J. Sci. Comput. 21 (2000) $2126-2143$.

[20] J.B. Keller, R.M. Lewis, Asymptotic methods for partial differential equations: the reduced wave equation and Maxwell's equations, Surveys Appl. Math. 1 (1995) 1-82.

[21] S. Kim, R. Cook, 3-D traveltime computation using second-order ENO scheme, Geophysics 64 (1999) 1867-1876.

[22] P.L. Lions, Generalized Solutions of Hamilton-Jacobi Equations, Pitman Advanced Publishing Program, Boston, 1982.

[23] Z. Liu, N. Bleistein, Migration velocity analysis: theory and an iterative algorithm, Geophysics 60 (1995) 142-153.

[24] M.J.P. Musgrave, Crystal Acoustics, Holden-Day, San Francisco, 1970.

[25] S.J. Osher, A level set formulation for the solution of the Dirichlet problem for Hamilton-Jacobi equations, SIAM J. Math. Anal. 24 (1993) $1145-1152$.

[26] S.J. Osher, L.-T. Cheng, M. Kang, H. Shim, Y.-H. Tsai, Geometrical optics in a phase space based level set and Eulerian framework, J. Comput. Phys. 179 (2002) 622-648.

[27] S.J. Osher, J.A. Sethian, Fronts propagating with curvature dependent speed: algorithms based on Hamilton-Jacobi formulations, J. Comput. Phys. 79 (1988) 12-49.

[28] S.J. Osher, C.W. Shu, High-order essentially nonoscillatory schemes for Hamilton-Jacobi equations, SIAM J. Numer. Anal. 28 (1991) 907-922.

[29] D. Peng, B. Merriman, S. Osher, H.K. Zhao, M. Kang, A PDE-based fast local level set method, J. Comput. Phys. 155 (1999) $410-438$.

[30] V. Pereyra, Two-point ray tracing in general 3-D media, Geophys. Prosp. 40 (1992) 267-287.

[31] J. Qian, W.W. Symes, Paraxial eikonal solvers for anisotropic quasi-P traveltimes, J. Comput. Phys. 173 (2001) 1-23.

[32] J. Qian, W.W. Symes, Adaptive finite difference method for traveltime and amplitude, Geophysics 67 (2002) 167-176.

[33] J. Qian, W.W. Symes, Finite-difference quasi-P traveltimes for anisotropic media, Geophysics 67 (2002) 147-155.

[34] J. Qian, W.W. Symes, Paraxial geometrical optics for quasi-P waves: theories and numerical methods, Wave Motion 35 (2002) $205-221$.

[35] J. Qian, W.W. Symes, A paraxial formulation for the viscosity solution of quasi-P eikonal equations, Comput. Math. Appl., in press.

[36] S.J. Ruuth, B. Merriman, S.J. Osher, A fixed grid method for capturing the motion of self-intersecting interfaces and related PDEs, J. Comput. Phys. 151 (1999) 836-861.

[37] W.A. Schneider Jr., K. Ranzinger, A. Balch, C. Kruse, A dynamic programming approach to first arrival traveltime computation in media with arbitrarily distributed velocities, Geophysics 57 (1992) 39-50.

[38] J.A. Sethian, A.M. Popovici, 3-D traveltime computation using the fast marching method, Geophysics 64 (1999) 516-523.

[39] J.A. Sethian, A. Vladimirsky, Ordered upwind methods for static Hamilton-Jacobi equations: theory and algorithms, PAM-792, University of California at Berkeley, Berkeley, CA, 2001.

[40] R.J. Spiteri, S.J. Ruuth, A new class of optimal high-order strong-stability-preserving time discretization methods, SIAM J. Numer. Anal. 40 (2) (2002) 469-491.

[41] J. Steinhoff, M. Fan, L. Wang, A new Eulerian method for the computation of propagating short acoustic and electromagnetic pulses, J. Comput. Phys. 157 (2000) 683-706.

[42] W.W. Symes, A slowness matching finite difference method for traveltimes beyond transmission caustics, in: Expanded Abstracts of 68th Annual Internat. Mtg., Soc. Expl. Geophys. 1945-1948 (1998), Soc. Expl. Geophys., Tulsa, OK, 1998.

[43] W.W. Symes, J.J. Carazzone, Velocity inversion by differential semblance optimization, Geophysics 56 (1991) $654-663$.

[44] W.W. Symes, J. Qian, A slowness matching Eulerian method for multivalued solutions of eikonal equations, J. Sci. Comput., in press.

[45] W.W. Symes, R. Versteeg, A. Sei, Q.H. Tran, Kirchhoff simulation, migration and inversion using finite difference traveltimes and amplitudes, Annual Report, The Rice Inversion Project, Rice University, 1994. http://www.trip.caam.rice.edu/.

[46] L. Thomsen, Weak elastic anisotropy, Geophysics 51 (1986) 1954-1966.

[47] I. Tsvankin, L. Thomsen, Inversion of reflection traveltimes for transverse isotropy, Geophysics 60 (1995) $1095-1107$.

[48] J. van Trier, W.W. Symes, Upwind finite-difference calculations of traveltimes, Geophysics 56 (1991) 812-821.

[49] J. Vidale, Finite-difference calculation of travel times, Bull. Seis. Soc. Am. 78 (1988) 2062-2076.

[50] V. Vinje, E. Iversen, H. Gjystdal, Traveltime and amplitude estimation using wavefront construction, Geophysics 58 (1993) $1157-1166$.

[51] B.S. White, The stochastic caustic, SIAM J. Appl. Math. 44 (1984) 127-149. 